\title{
Histopathological analysis of the progression pattern of subungual melanoma: late tendency of dermal invasion in the nail matrix area
}

\author{
Hyun-Tae Shin ${ }^{1,5}$, Kee-Taek Jang ${ }^{2,5}$, Goo-Hyun Mun ${ }^{3}$, Dong-Youn Lee ${ }^{1}$ and Jason B Lee ${ }^{4}$ \\ ${ }^{1}$ Department of Dermatology, Samsung Medical Center, Sungkyunkwan University School of Medicine, \\ Seoul, Republic of Korea; ${ }^{2}$ Department of Pathology, Samsung Medical Center, Sungkyunkwan University \\ School of Medicine, Seoul, Republic of Korea; ${ }^{3}$ Department of Plastic Surgery, Samsung Medical Center, \\ Sungkyunkwan University School of Medicine, Seoul, Republic of Korea and ${ }^{4}$ Department of Dermatology \\ and Cutaneous Biology, Thomas Jefferson University Hospital, Philadelphia, PA, USA
}

\begin{abstract}
Subungual melanoma is a rare subtype of melanoma that usually originates and spreads from the nail matrix. Because of its poor prognosis and short matrix-to-bone distance, amputation has been traditionally performed. Recently, conservative surgery has been attempted for early subungual melanoma, but the evidence supporting this practice is sparse. As little is known about the progression pattern of subungual melanoma, further advances on the subject may provide better guidance on the optimal surgical approach. Histopathology slides, clinical records, and photographs of $\mathbf{2 3}$ cases of subungual melanoma were reviewed. For all cases, each area of the nail unit-proximal nail fold, nail matrix, nail bed, and/or hyponychium-in longitudinal sections was available for histological examination. Growth pattern, dermal invasion, and thickness were assessed in each area of the nail unit. There were five cases of melanoma in situ. Eighteen cases showed dermal invasion in at least one area of the nail unit. There were no cases showing dermal invasion in the nail matrix area only. In four cases, dermal invasion involved areas of the nail unit other than the nail matrix. In 14 cases, dermal invasion involved the nail matrix area as well as other areas of the nail unit. Except for one case, the nail matrix area showed thinner dermal invasion compared with dermal invasion in other areas of the nail unit. In conclusion, dermal invasion of subungual melanoma in the nail matrix area tends to occur later than other areas of the nail unit. Longitudinal incisional biopsy is necessary to accurately evaluate melanoma invasion. The findings of this study suggest that conservative surgical treatment for early subungual melanoma may be justified as the nail matrix area, an area of thin dermis and close proximity to the underlying bone, appears to be more resistant to invasion.
\end{abstract}

Modern Pathology (2014) 27, 1461-1467; doi:10.1038/modpathol.2014.65; published online 18 April 2014

Keywords: dermal invasion; nail matrix; subungual melanoma

The normal nail unit has unique anatomical structures, which consist of proximal nail fold, nail matrix, nail bed, and hyponychium (Figure 1). Beneath the nail matrix and nail bed, there exists specialized nail mesenchyme, referred to as onychodermis, ${ }^{1}$ associated with attenuated subcutaneous fat. When comparing distances from the different areas of the nail unit to the phalangeal bone, it is the

Correspondence: Professor D-Y Lee, MD, PhD, Department of Dermatology, Samsung Medical Center, Sungkyunkwan University School of Medicine, Seoul, 135-710, Republic of Korea.

E-mail: dylee@skku.edu

${ }^{5}$ Two authors contributed equally to this study.

Received 8 October 2013; revised 7 January 2014; accepted 7 January 2014; published online 18 April 2014 shortest from the nail matrix area. The traditional aggressive surgical approach to subungual melanoma has been due in part to this short matrixto-bone distance.

Subungual melanoma is a rare variant of melanoma, frequently misdiagnosed clinically and histopathologically in its early stages. ${ }^{2}$ The delay in diagnosis often results in late-stage disease that is associated with poor prognosis, usually requiring amputation of the affected digit. ${ }^{3,4}$ Recently, there have been several reports of non-amputative conservative treatment for in situ or thin lesions of subungual melanoma. ${ }^{5-7}$ Subungual melanoma usually originates from the nail matrix and may spread to the surrounding nail bed, hyponychium, and proximal nail fold (Figure 1)., ${ }^{2,8}$ Little is known about the 
progression pattern of subungual melanoma and the rate of dermal invasion in each area of the nail unit. Previously, Izumi et $a l^{8}$ reported that in early subungual melanoma, proliferation of the atypical melanocytes was more prominent in the hyponychium than the nail bed or nail matrix. If dermal invasion occurs more frequently in the nail matrix, an area with the shortest distance to the bone within the nail unit, conservative treatment may not be appropriate for early stages of subungual melanoma. ${ }^{9}$ In this retrospective investigation, the growth pattern, dermal invasion, and thickness of subungual melanoma in each area of the nail unit were analyzed to better understand the progression pattern of subungual melanoma with the aim of providing some basis for the different surgical approaches.

\section{Material and methods}

After receiving approval from the institutional review board, 56 cases of subungual melanoma diagnosed at the Samsung Medical Center from 1997 to 2012 were identified and reviewed, which included histology slides, clinical records, and photographs of the cases. After the review of the histological sections, the cases that did not contain tissue samples from the nail matrix and nail bed were excluded. Finally, total 23 cases, which had tissue samples from each area of the nail unit (proximal nail fold, nail matrix, nail bed, and/or hyponychium), were selected for this study.

Clinical data about sex, age, onset time of disease, trauma history, site of primary lesion, clinical presentation, presence of distant metastasis, treatment, and prognosis were obtained from the electronic medical records and photographs.

When examining histological sections, the growth pattern and presence of dermal invasion of subungual melanoma in each area of the nail unit were

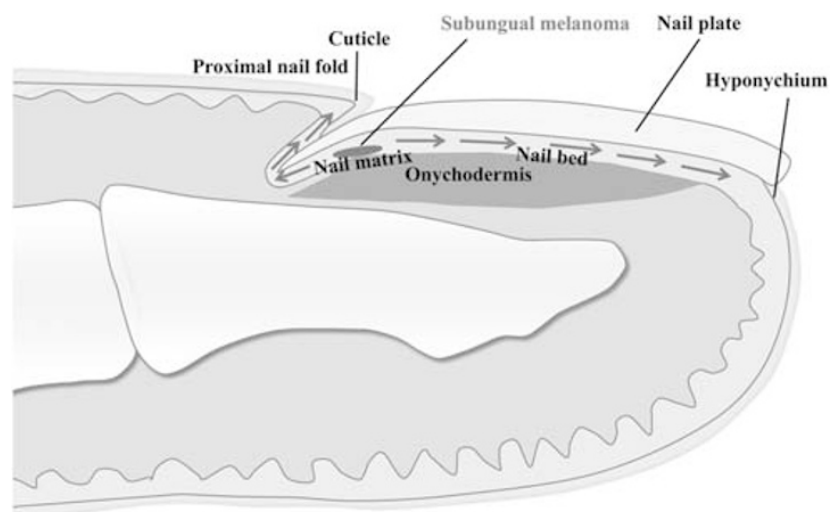

Figure 1 Simplified diagram of the longitudinal section of the nail unit. Subungual melanoma usually originates from nail matrix and spreads to the surrounding areas such as nail bed, hyponychium, and proximal nail fold. noted. If there was dermal invasion in the proximal nail fold or hyponychium, the tumor thickness from the top of granular layer or the base of ulceration to deepest melanoma front was measured (Figure $4 \mathrm{~d}$ ). In the nail matrix and nail bed, it was measured from the junction between epithelium and nail plate to deepest melanoma front. The frequency of dermal invasion in each area of the nail unit then was tabulated. When the presence of dermal invasion was difficult to determine, serial sections or immunohistochemical staining including HMB-45 (1:50 dilution; Dako, Carpinteria, CA, USA) and/or Melan-A (1:50 dilution; Dako) were performed to identify the dermal invasion. Amino-3-ethyl-9-carbozole was used as a chromogen for immunohistochemical staining.

Data were compared using $t$-test. Statistical analysis was performed with SPSS software version 19.0 (SPSS Inc.,Chicago, IL). All tests were assessed at $\alpha=0.05$ significant level. $P<0.05$ is considered to be statistically significant. All values were rounded in the second decimal place.

\section{Results}

\section{Clinical Features}

Of the 23 cases of subungual melanoma, 9 (39\%) cases were men and 14 (61\%) were women. Mean age at the diagnosis was 56.5 years (28 to 82 years).

Table 1 Clinical features of subungual melanoma cases

\begin{tabular}{lc}
\hline Features & $\begin{array}{c}\text { Number of cases } \\
\text { with available data }\end{array}$ \\
\hline Sex (number) & 23 \\
Male & $9(39 \%)$ \\
Female & $14(61 \%)$ \\
Age (years) & 23 \\
Mean age & 56.5 \\
Range & $28-82$ \\
Onset (years ago) & \\
Mean onset & 20 \\
Range & 5.2 \\
Trauma history (number) & $0.6-15$ \\
Yes & 23 \\
No or not mentioned & $6(26 \%)$ \\
Site of primary lesion (number) & $17(74 \%)$ \\
Finger & \\
Thumb & 23 \\
Index & $16(70 \%)$ \\
Middle & $10(43 \%)$ \\
Ring & $3(13 \%)$ \\
Great & $2(9 \%)$ \\
Initial clinical presentation & $1(4 \%)$ \\
Longitudinal melanonychia & $7(30 \%)$ \\
\hline
\end{tabular}



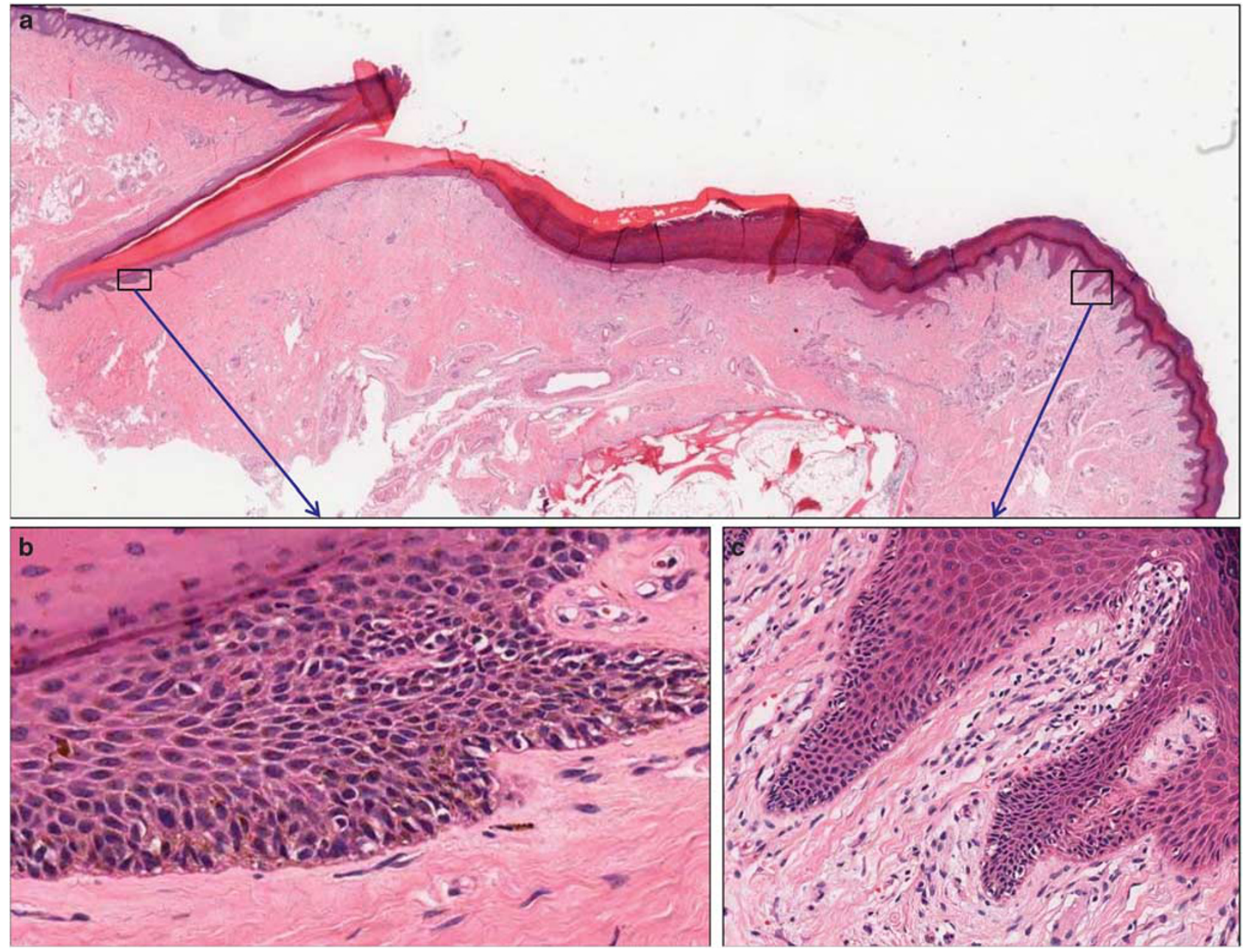

Figure 2 Histopathological findings of subungual melanoma in situ (case 5). (a) Longitudinal section of the nail unit. (b) Proliferation of atypical melanocytes in the basal layer and suprabasal layers of the nail matrix. (c) Proliferation of atypical melanocytes along the basal layer of the hyponychium.

Mean duration of the disease was 5.2 years ( 0.6 to 15 years). Six cases (26\%) had a trauma history and 17 cases $(74 \%)$ did not have a trauma history or history information was not available. Sixteen cases $(70 \%)$ occurred on the fingers, whereas 7 cases $(30 \%)$ occurred on the toes. Most common site was the thumb (10 cases, 43\%), followed by the great toe ( 7 cases, 30\%), the index finger ( 3 cases, 13\%), the middle finger ( 2 cases, $9 \%$ ), and the ring finger (1 case, 4\%). Initial clinical presentation of the 20 cases, in which data were available, was longitudinal melanonychia. The clinical features of 23 cases of subungual melanoma are summarized in Table 1.

\section{Histopathological Findings of Each Part of the Nail Unit}

Five cases (case 1-5) didn't show dermal invasion in any part of the nail unit (Figure 2), representing in situ lesions. Eighteen cases (case 6-23) showed dermal invasion in at least one area of the nail unit. Among the cases with dermal invasion, there were no cases that showed dermal invasion in the nail matrix area only. Four cases (case 6-9) showed dermal invasion in areas of the nail unit other than the nail matrix (Figure 3). In 14 cases, dermal invasion involved the nail matrix (case 10-23) as well as one or more other areas of the nail unit (Figure 4). Except case 17, all cases $(n=13)$ with dermal invasion, which involved the nail matrix area, showed thinner tumor thickness in the nail matrix compared with other areas of the nail unit with dermal invasion. The mean tumor thickness of cases in which dermal invasion did not involve the matrix was $2.2 \pm 2.0 \mathrm{~mm}$ (case $6-9$ ) whereas cases in which dermal invasion involved the matrix was $2.9 \pm 2.3 \mathrm{~mm}$ (cases 10-23). However, this difference of the tumor thickness was not statistically significant $(P>0.05)$. Band-like proliferation of atypical melanocytes (a stack of atypical melanocytes) in the 

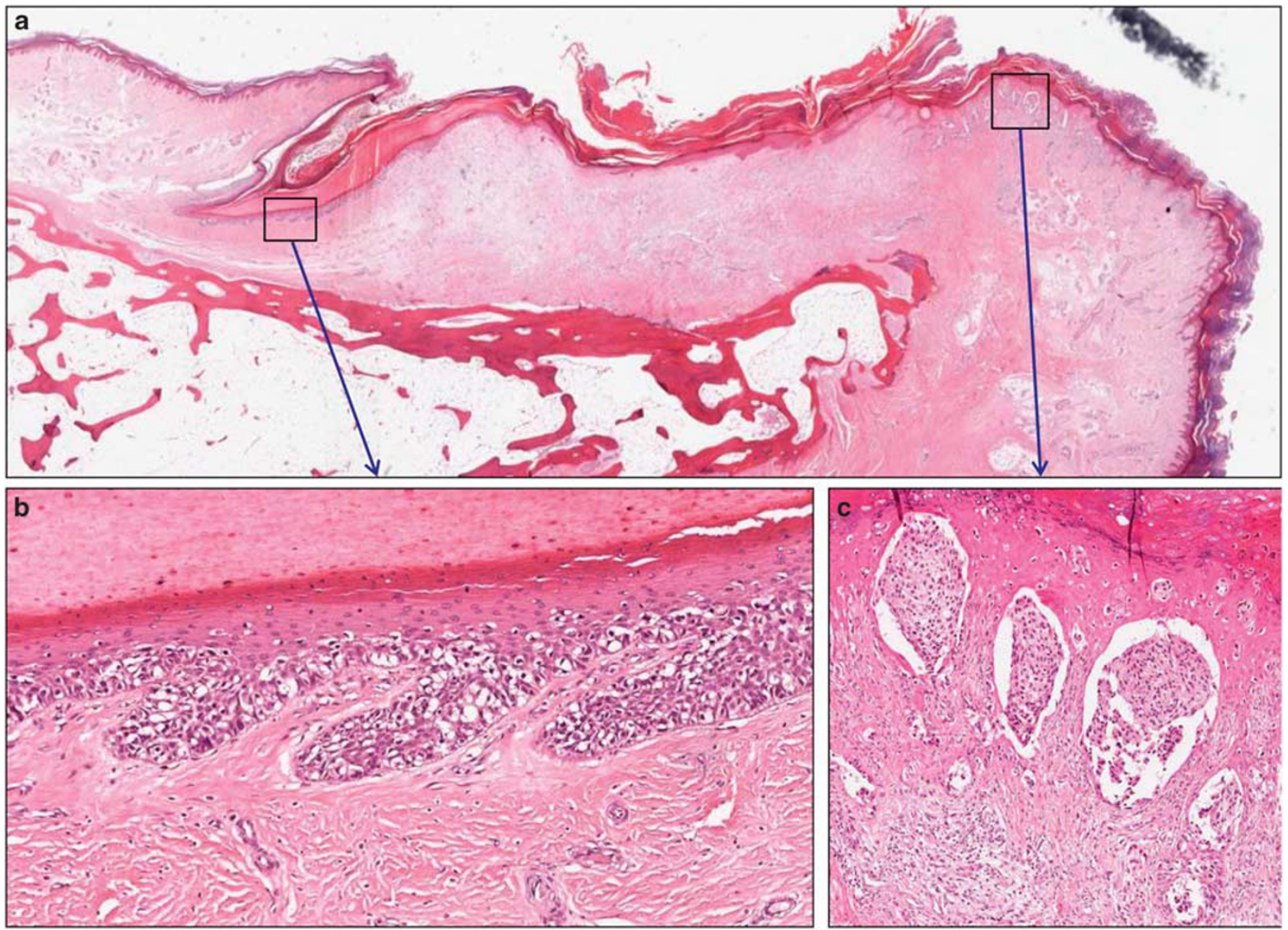

Figure 3 Histopathological findings of invasive subungual melanoma (case 7). (a) Longitudinal section of the nail unit. (b) Stacking of atypical melanocytes in the nail matrix. (c) Dermal invasion of atypical melnaocytes in nests and solitary units in the hyponychium; note the striking variation in size and shape of the nests.

nail matrix, especially within the distal region of the matrix, was observed in $70 \%(16 / 23)$ of the cases. Histopathological findings in each area of the nail unit, status of metastasis, treatment method, and duration of follow-up are summarized in Table 2. Six cases (case 9, 19-23) with distant metastasis had ipsilateral axillary or inguinal lymph node metastasis.

\section{Surgery and Prognosis of Subungual Melanoma}

Three cases with melanoma in situ (case 2-4) and 2 cases with dermal invasion (case 10 and 16) underwent conservative surgery (excision of whole nail structures and free flap), whereas 17 cases underwent amputation. Case 14 did not receive any surgery. The mean follow-up period of the cases with the conservative surgery was 8.8 months and that of amputation was 32.4 months. There was no local recurrence after surgery. Only one patient (case 9) received palliative treatment and died of multiple distant metastases at 20 months after amputation.

\section{Discussion}

In nearly all cases, subungual melanoma arises from the nail matrix, although rarely it may arise from the nail bed theoretically from the residing quiescent melanocytes. ${ }^{2,10}$ When subungual melanoma originates from the nail matrix, linear pigmentation of the nail plate (longitudinal melanonychia) is the most common clinical presentation, accounting for up to $76 \%$ of the cases. ${ }^{10}$ As the melanoma from the nail matrix spreads to other parts of the nail unit, pigmentation of the nail fold and/or the hyponychium (Hutchinson's sign) and nail dystrophy may be observed. ${ }^{11}$ In this study, the majority of the cases (20/23) presented with longitudinal melanonychia, indicative of nail matrix being the site of origin. The clinical history was not available for the remaining three cases, but in each of these cases, melanoma involved the nail matrix as well as other areas of the nail unit, suggesting that the nail matrix was also their site of origin. When subungual melanoma originates from the nail bed, it typically presents as a non-pigmented subungual nodule usually 


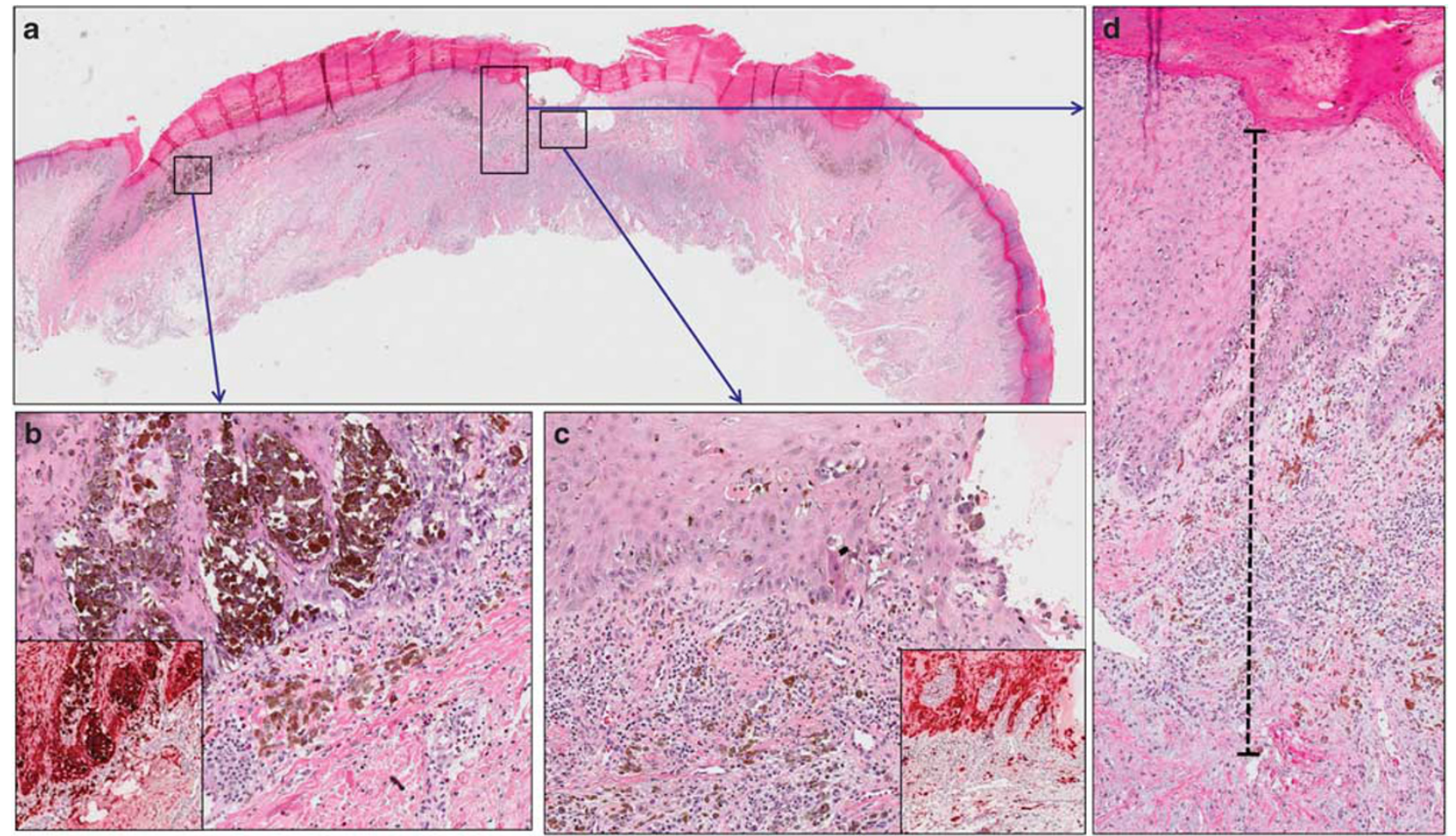

Figure 4 Histopathological findings of invasive subungual melanoma (case 14). (a) Longitudinal section of the nail unit. (b) HMB-45 immunostain highlights the nested atypical melanocytes in the nail matrix and upper dermis (inset). (c) HMB-45 immunostain highlights the solitary atypical melanocytes in the nail bed and upper dermis (inset). (d) The dotted line indicates tumor thickness from the junction between epithelium and nail plate to deepest melanoma front in the nail bed area.

accompanied by onycholysis. ${ }^{10}$ As the subungual nodule may represent the thicker portion of melanoma that has originated from the matrix, the lack of involvement of the nail matrix requires histologic confirmation.

As the only significant predictor of prognosis, the thickness of melanoma is one of the most important staging criteria. ${ }^{2,3,8,12}$ Unlike the rest of the skin, the complex anatomy of the nail apparatus poses some challenges in determining the depth of subungual melanoma accurately, which may explain why there is no consensus on the method of determining the thickness of subungual melanoma. ${ }^{2}$ Because the thickness of epidermis in each area of normal nail unit varies, dermal invasion may be overestimated depending on where the melanoma is measured. Furthermore, subungual melanomas are frequently associated with epidermal hyperplasia, ${ }^{2,8}$ a factor that may also lead to overestimation of the actual dermal invasion. ${ }^{2}$ Moreover, Breslow's method of assessing thickness of subungual melanomas may be inappropriate as, unlike other cutaneous sites, the granular layer in the nail matrix and nail bed is absent. Therefore, accurate and reproducible assessment of dermal invasion of subungual melanoma is limited by its unique anatomy. Finally, in the nail unit, not only the subcutaneous layer is scant or absent, but also the anatomic boundary between the papillary dermis and reticular dermis may not be discernible. For these reasons, accurate assessment of Clark level is also problematic in subungual melanoma. As thickness is an important prognostic predictor of subungual melanoma, standardized thickness measurement of subungual melanoma that takes into account the unique anatomic features of the nail unit is needed. Measuring from the highest point of the viable epithelium to the deepest melanoma front by dermatopathologists or pathologists who are familiar with the anatomy of the nail unit may offer an accurate and reproducible method of measurement. Alternatively, measuring from the dermoepithelial junction to the greatest depth of the melanoma may be considered. The latter method may offer the most accurate measurement of dermal invasion that accounts for the variable thickness of the epithelium in the nail unit.

Similar to other types of melanoma, subungual melanoma grows radially at early stages with dermal invasion occurring at more advanced stages. , $^{2,13}$ In this study, although all cases seemed to originate from the nail matrix, there were no cases with dermal invasion that involved only the nail matrix and four cases with dermal invasion involved areas of the nail unit other than the nail matrix area. When dermal invasion occurred in the nail matrix as well as other areas of the nail unit, the tumor in other 
Table 2 Histopathological findings of each part of the nail unit, status of metastasis, treatment method, and duration of follow-up

\begin{tabular}{|c|c|c|c|c|c|c|c|}
\hline Case no. & $\begin{array}{l}\text { Proximal } \\
\text { nail fold } \\
\text { (depth }(\mathrm{mm}))\end{array}$ & $\begin{array}{l}\text { Nail matrix } \\
(\text { depth }(\mathrm{mm}))\end{array}$ & $\begin{array}{l}\text { Nail bed } \\
(\text { depth }(\mathrm{mm}))\end{array}$ & $\begin{array}{l}\text { Hyponychium } \\
\text { (depth }(\mathrm{mm}) \text { ) }\end{array}$ & Distant metastasis & Surgical methods & $\begin{array}{l}\text { Post- } \\
\text { operative } \\
\text { follow-up } \\
\text { (months) }\end{array}$ \\
\hline 1 & In situ & In situ & In situ & Normal & $(-)$ & Amputation & A\&W 36 \\
\hline 2 & In situ & In situ & In situ & In situ & $(-)$ & Conservative treatment & A\&W 12 \\
\hline 3 & In situ & In situ & In situ & In situ & $(-)$ & Conservative treatment & $\mathrm{A} \& \mathrm{~W} 6$ \\
\hline 4 & In situ & In situ & In situ & In situ & $(-)$ & Conservative treatment & $\mathrm{A} \& \mathrm{~W} 1$ \\
\hline 5 & In situ & In situ & In situ & In situ & $(-)$ & Amputation & A\&W 32 \\
\hline 6 & Invasion (0.2) & In situ & In situ & NA & $(-)$ & Amputation & A\&W 4 \\
\hline 7 & Invasion (0.2) & In situ & Invasion (0.2) & Invasion (1.6) & $(-)$ & Amputation & A\&W 58 \\
\hline 8 & In situ & In situ & Invasion (2.1) & In situ & $(-)$ & Amputation & $\mathrm{A} \& \mathrm{~W} 60$ \\
\hline 9 & In situ & In situ & Invasion (5.0) & Invasion (3.7) & $\begin{array}{l}\text { Ipsilateral axillary } \\
\text { lymph node, bone }\end{array}$ & Amputation & 20, Death \\
\hline 10 & In situ & Invasion (0.2) & Invasion (0.4) & Invasion $(0.3)$ & $(-)$ & Conservative treatment & A\&W 24 \\
\hline 11 & In situ & Invasion $(0.2)$ & Invasion (0.4) & In situ & $(-)$ & Amputation & A\&W 58 \\
\hline 12 & In situ & Invasion (0.1) & Invasion (0.4) & Invasion (0.3) & $(-)$ & Amputation & A\&W 8 \\
\hline 13 & Invasion (1.0) & Invasion (0.2) & Invasion (0.2) & invasion (0.3) & $(-)$ & Amputation & A\&W 72 \\
\hline 14 & Invasion (0.5) & Invasion (0.8) & Invasion (1.3) & Invasion (0.8) & $\mathrm{NA}$ & NA & NA \\
\hline 15 & Invasion (0.7) & Invasion (0.6) & Invasion (2.0) & Invasion (0.4) & $(-)$ & Amputation & A\&W 41 \\
\hline 16 & Invasion (0.4) & Invasion (1.0) & Invasion (2.3) & Invasion (0.6) & $(-)$ & Conservative treatment & $\mathrm{A} \& W 1$ \\
\hline 17 & Invasion (0.5) & Invasion (2.7) & Invasion (1.2) & Invasion (0.4) & $(-)$ & Amputation & $\mathrm{A} \& \mathrm{~W} 8$ \\
\hline 18 & In situ & Invasion (1.8) & Invasion (4.5) & In situ & $(-)$ & Amputation & A\&W 18 \\
\hline 19 & In situ & Invasion (0.5) & Invasion (2.2) & Invasion (0.9) & $\begin{array}{l}\text { Ipsilateral axillary } \\
\text { lymph node }\end{array}$ & Amputation & A\&W 18 \\
\hline 20 & Invasion (3.2) & Invasion (1.1) & Invasion (3.7) & Normal & $\begin{array}{l}\text { Ipsilateral axillary } \\
\text { lymph node, soft tissue }\end{array}$ & Amputation & A\&W 61 \\
\hline 21 & Invasion (0.1) & Invasion (3.0) & Invasion (5.6) & NA & $\begin{array}{l}\text { Ipsilateral axillary } \\
\text { lymph node, lung }\end{array}$ & Amputation & A\&W 27 \\
\hline 22 & Invasion (1.0) & Invasion (4.1) & Invasion (6.7) & Invasion (3.3) & $\begin{array}{l}\text { Ipsilateral inguinal } \\
\text { lymph node, soft tissue }\end{array}$ & Amputation, & A\&W 12 \\
\hline 23 & Invasion (1.0) & Invasion (3.6) & Invasion (7.0) & Invasion (1.0) & $\begin{array}{l}\text { Ipsilateral inguinal } \\
\text { lymph node }\end{array}$ & Amputation & A\&W 18 \\
\hline
\end{tabular}

Abbreviations: A\&W, alive and well; NA, not available.

areas of the nail unit was thicker than that in the nail matrix area. In cases with dermal invasion that involved nail matrix as well as other parts of the nail unit, the mean thickness was greater compared with the cases with dermal invasion that did not involve the matrix. The difference between the two groups, however, was not statistically significant. Taken together, these findings suggest that dermal invasion in the nail matrix may occur in later stages of subungual melanoma progression than that in other parts of the nail unit. In addition, these results further support the recommendation of longitudinal incisional biopsy that includes all parts of the nail unit when subungual melanoma is strongly suspected clinically, as it would offer the most optimal method in evaluating dermal invasion and thickness of subungual melanoma accurately. The routine practice of sampling only the nail matrix area may underestimate the depth of the melanoma according to the findings of this study.

Nail matrix is a germinative and proliferative compartment for nail plate formation. ${ }^{14,15}$ The late tendency of dermal invasion in nail matrix area may be explained by the possibility that upward growth of nail matrix cells delays dermal invasion of subungual melanoma in the nail matrix. In the nail matrix, $\alpha 2, \alpha 3$, and $\beta 1$ integrins are expressed not only in the basal layer but also in the suprabasal layers, unlike their expression just in the basal layer of the other parts of epithelium. ${ }^{16}$ This different expression of adhesion molecules may result in intimate adhesion of melanocyte with surrounding keratinocyte, mediating the late dermal invasion of subungual melanoma in the nail matrix area.

Sixteen cases $(70 \%)$ of our study showed stacking of atypical melanocytes in the suprabasal layers of the nail matrix, with the distal matrix displaying more pronounced stacking pattern compared with the proximal matrix. The melanocytes in the nail matrix were distributed throughout the basal layer and suprabasally in solitary units and small clusters. ${ }^{16}$ Because there are many more active melanocytes (DOPA-positive) in the distal matrix than the proximal matrix, ${ }^{17}$ longitudinal melanonychia and subungual melanoma are believed to originate more frequently in the distal matrix. The relative large number of active melanocytes in the distal matrix may account for the more prominent stacking of atypical melanocytes and the late tendency of dermal invasion of subungual melanoma in the nail matrix.

Amputation at the distal phalangeal joint is generally regarded as the surgical treatment of choice for subungual melanoma. ${ }^{4}$ Recently, however, some authors have suggested a non-amputative conservative treatment of subungual melanoma to preserve the phalangeal bone for in situ or thin lesions of subungual melanoma. ${ }^{5,6,18-20}$ Few publications provide 
evidence of safety and effectiveness of conservative treatment of early subungual melanoma, although several cases of recurrences after conservative treatment have been reported in the literature. ${ }^{5,6}$ Generally, deep margin of cutaneous melanoma is confined above the underlying subcutaneous tissue. ${ }^{21-23}$ The dermis below the nail matrix is thin with minimal to absent subdermal fat. ${ }^{9,15}$ Because subungual melanoma usually originates from the matrix where dermis is thin and in close proximity to the underlying bone, a surgeon may have concerns about conservative surgery for early subungual melanoma. ${ }^{9}$ The relative resistance to dermal invasion within the nail matrix observed in this study, however, provides some support for non-amputative conservative surgery in early subungual melanoma.

The limitations of this study include the relatively small sample size and the short follow-up period, which was not sufficient to compare the safety of conservative surgery with amputation. This study, however, is unique in that only those cases that had histological sections from the different areas of the nail unit were included, which allowed for comparing dermal invasion pattern of the different areas.

In conclusion, the results of this study suggest that dermal invasion in the nail matrix occurs at later stages of subungual melanoma progression. Longitudinal incisional biopsy is necessary to accurately evaluate melanoma invasion, as variation in the thickness may be observed in the different areas of the nail unit. The progression pattern of subungual melanomas observed in this study lends support to non-amputative conservative surgery for cases of early subungual melanomas.

\section{Disclosure/conflict of interest}

The authors declare no conflict of interest.

\section{References}

1 Lee DY, Park JH, Shin HT, et al. The presence and localization of onychodermis (specialized nail mesenchyme) containing onychofibroblasts in the nail unit: a morphological and immunohistochemical study. Histopathology 2012;61:123-130.

2 Tan KB, Moncrieff M, Thompson JF, et al. Subungual melanoma: a study of 124 cases highlighting features of early lesions, potential pitfalls in diagnosis, and guidelines for histologic reporting. Am J Surg Pathol 2007;31:1902-1912.

3 Banfield CC, Redburn JC, Dawber RP. The incidence and prognosis of nail apparatus melanoma. A retrospective study of 105 patients in four English regions. Br J Dermatol 1998;139:276-279.

4 O'Leary JA, Berend KR, Johnson JL, et al. Subungual melanoma. A review of 93 cases with identification of prognostic variables. Clin Orthop Relat Res 2000; 206-212.
5 Sureda N, Phan A, Poulalhon N, et al. Conservative surgical management of subungual (matrix derived) melanoma: report of seven cases and literature review. Br J Dermatol 2011;165:852-858.

6 Neczyporenko F, Andre J, Torosian K, et al. Management of in situ melanoma of the nail apparatus with functional surgery: report of 11 cases and review of the literature. J Eur Acad Dermatol Venereol 2013; In press.

7 Levine SM, Shapiro RL. Surgical treatment of malignant melanoma: practical guidelines. Dermatol Clin 2012;30:487-501.

8 Izumi M, Ohara K, Hoashi T, et al. Subungual melanoma: histological examination of 50 cases from early stage to bone invasion. J Dermatol 2008;35: 695-703.

9 Kim JY, Jung HJ, Lee WJ, et al. Is the distance enough to eradicate in situ or early invasive subungual melanoma by wide local excision? from the point of view of matrix-to-bone distance for safe inferior surgical margin in Koreans. Dermatology 2011;223:122-123.

10 Tosti A, Richert B, Pazzaglia M. Tumors of the nail apparatus, In: Scher RK, Daniel CR (eds). Nails: Diagnosis, Therapy, Surgery, 3rd edn. Elsevier Saunders: Oxford, UK; 2005, pp 202-203.

11 Ruben BS. Pigmented lesions of the nail unit: clinical and histopathologic features. Semin Cutan Med Surg 2010;29:148-158.

12 Blessing K, Kernohan NM, Park KG. Subungual malignant melanoma: clinicopathological features of 100 cases. Histopathology 1991;19:425-429.

13 Clark WH. Tumour progression and the nature of cancer. Br J Cancer 1991;64:631-644.

14 de Berker D, Angus B. Proliferative compartments in the normal nail unit. Br J Dermatol 1996;135:555-559.

15 Haneke E. Surgical anatomy of the nail apparatus; In: Richert B, Di Chiacchio N, Haneke E (eds). Nail Surgery. Informa Healthcare: New York, NY, USA; 2011, pp 1-10.

16 Tosti A, Cameli N, Piraccini BM, et al. Characterization of nail matrix melanocytes with anti-PEP1, anti-PEP8, TMH-1, and HMB-45 antibodies. J Am Acad Dermatol 1994;31:193-196.

17 Perrin C, Michiels JF, Pisani A, et al. Anatomic distribution of melanocytes in normal nail unit: an immunohistochemical investigation. Am J Dermatopathol 1997;19:462-467.

18 Moehrle M, Metzger S, Schippert W, et al. 'Functional' surgery in subungual melanoma. Dermatol Surg 2003;29:366-374.

19 Lazar A, Abimelec P, Dumontier C. Full thickness skin graft for nail unit reconstruction. J Hand Surg $\mathrm{Br}$ 2005;30:194-198.

20 Duarte AF, Correia O, Barros AM, et al. Nail matrix melanoma in situ: conservative surgical management. Dermatology 2010;220:173-175.

21 Kenady DE, Brown BW, McBride CM. Excision of underlying fascia with a primary malignant melanoma: effect on recurrence and survival rates. Surgery 1982;92:615-618.

22 Testori A, Rutkowski P, Marsden J, et al. Surgery and radiotherapy in the treatment of cutaneous melanoma. Ann Oncol 2009;20(Suppl 6):vi22-vi29.

23 Weinstein MC, Brodell RT, Bordeaux J, et al. The art and science of surgical margins for the dermatopathologist. Am J Dermatopathol 2012;34:737-745. 